\begin{tabular}{|c|c|c|}
\hline  & $\begin{array}{c}\text { European Journal of Chemistry } \\
\text { Journal homepage: } \underline{\text { www.eurjchem.com }}\end{array}$ & 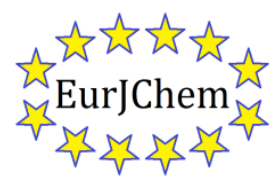 \\
\hline
\end{tabular}

\title{
Evaluation of global hardness of atoms based on the commonality in the basic philosophy of the origin and the operational significance of the electronegativity and the hardness. Part I. The Gordy's scale of electronegativity and the global hardness
}

\author{
Nazmul Islam and Dulal Chandra Ghosh* \\ Department of Chemistry, University of Kalyani, Kalyani-741235, India \\ ${ }^{*}$ Corresponding author at: Department of Chemistry, University of Kalyani, Kalyani-741235, India. Tel.: +91.9433389909; fax: +91.3325828282.

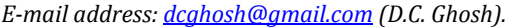

\section{ARTICLE INFORMATION}

Received: 31 March 2010

Received in revised form: 30 April 2010

Accepted: 30 April 2010

Online: 30 June 2010

\section{KEYWORDS}

Commonality between electronegativity and hardness

Gordy scale of electronegativity

Scale of global hardness based on potential

concept

Periodicity of global hardness of atoms

\section{Introduction}

The hardness and the electronegativity are two different old and extremely useful conceptual constructs of chemistry and physics. Although the meaning and purpose of the words 'hardness' and 'electronegativity' are intrinsically different and are used in different contexts of chemico-physical phenomenathe reactivity and stability of atoms, molecules and condensed matter, there are reasons to believe that there is much commonality in the basic philosophical structures relating to their origin and development. History shows that the term 'hardness' originated in solid state condensed matter physics signifying the resistance of a structure towards deformation [1]. Still there is another notion of hardness in chemistry, the chemical hardness, originating from the intrinsic resistance that manifests by holding the electron cloud tightly to the species- atoms, ions and molecules. The electron cloud of hard species does not deform on small perturbation of the chemical reaction of molecules. The idea of chemical hardness originated basically as a rationale of the hard-soft acid base theory [2,3]. Physicists and chemists have used the word 'hardness' virtually in different contexts and consequently the same word 'hardness' has two different connotations -'chemical hardness' and 'physical hardness'. However, the chemical hardness and physical hardness have fundamentally evolved with time to converge to the one and single unified concept the hardness in general for atoms [4]. However, the glimpses of similarities in the conceptual structures, and the mathematical formulae of evaluating the chemical and physical hardness can be found in the work of a good number of predecessor scientists [5-9].

Similarly, another very important qualitative theoretical construct in chemistry is electronegativity. This conceptual theoretical construct is indispensable of chemistry and physics as well. The electronegativity with its strong chemical organizing power has manifold use in chemistry as well as in physics for locating transfer, static and dynamic rearrangement of charge during chemico-physical processes. The electro negativity is not only ubiquitous in chemistry but also, in present times, finds application in physics, biology and geology [10-11]. It has also been pointed out that electronegativity idea helps in the qualitative understanding and elucidation of quark chemistry [12]. The concept has been extensively used in chemistry in correlating chemical binding, reactivity and many other physico-chemical properties of atoms and molecules over a long period of time. Coulson [13] and Fukui [14], the doyens of theoretical chemistry, pointed out the importance of the concept of electronegativity in elucidating and sketching the static distribution and dynamic rearrangement of electronic charge in molecules. The bond energies, bond polarities, dipole moments, force constants, and inductive effects etc are some conceptual descriptors of the real world that add to the essential understanding, rationalization and modeling of organic, inorganic and physical chemistry. Such fundamental descriptors can only be conceived in terms of the idea of electronegativity.

It is stated above that the hardness, as conceived in chemistry, signifies the resistance towards the deformation of 
charge cloud of chemical systems under small perturbation encountered during chemical processes. The general operational significance of the hard-soft chemical species may be understood in the following statement. If the electron cloud is strongly held by the nucleus, the chemical species is 'hard' but if the electron cloud is loosely held by the nucleus, the system is 'soft' [2,15-16].

In chemical domain, there is extensive theoretical search to quantify chemical hardness. The quest for the theoretical basis of the hard soft acid base behaviour has given birth of a new branch of theoretical chemistry known as 'Conceptual Density Functional Theory' CDFT [17-23] within the basic paradigm of density functional theory. The conceptual density functional theory has added (i) Maximum Hardness Principle, (MHP) [2425], and (ii) Minimum Polarizability Principle, (MPP) [26] to the list of the fundamental laws of nature. The conceptual density functional theory has been successfully exploited in elucidating and correlating mechanistic aspects viz. regioselectivity, catalysis, aromaticity, intramolecular rotation, inversion and isomerization reaction [27-34].

\subsection{Fundamental nature of hardness and electronegativity}

It is the experience of chemists and physicists that the principles of hardness and electronegativity work in chemistry and physics but they are not physical observables. Although, Putz [32] has discussed at length the question whether the electronegativity may be considered as quantum observable, quantum mechanics does not suggest operators whose eigen values are the electronegativity and hardness. One may find the resemblance between the appearance and significance of heuristically developed concepts of electronegativity and hardness in chemistry and physics and the unicorns of mythical saga [36]. They exist but never seen. Without the concept and operational significance of hardness and electronegativity, chemistry and many aspects of condensed matter physics becomes chaotic and the long established unique order in chemico-physical world would be disturbed. We may refer to the opinion of Parr et al. [37] who seem to have connected the reality of the hardness and the electronegativity with the noumenon of Kantian philosophy. The noumenon is an object knowable by the mind or intellect, not by the senses. Thus both the hardness and electronegativity are objects of purely intellectual intuition. We feel it pertinent to recall the opinion of Ayers [38] that before any algorithm of computing the hardness and the electronegativity is developed, the reification of abstract concept into things of the real world is necessary.

In a recent work, we have discussed the basic nature and definition of the hardness and the electronegativity from qualitative standpoint and discovered that the two different descriptors converge into one unifying principle [4]. But there is a claim by the adherents and proponents of density functional theory that the electronegativity and hardness have been elevated and sublimated from their qualitative ignominy to sound quantum mechanical basis with the help of density functional theory. The density functional ramification of the electronegativity and the hardness is an animated field of current research. It would be appropriate, therefore, to dwell upon the density functional underpinning of the electronegativity and the hardness to reveal the actual scientific status of the electronegativity and the hardness as conferred by the density functional theory.

Given the electron density function $\rho(\mathrm{r})$ in a chemical system (atom or molecule) and the energy functional $\mathrm{E}(\rho)$, the chemical potential, $\mu$ of that system in equilibrium has been defined as the derivative of the energy with respect to the number of electrons at fixed molecular geometry.

The chemical potential [36], $\mu$, is given by

$\mu=[\partial \mathrm{E} / \partial \mathrm{N}]_{v}$
Here $\mathrm{E}$ is expressed as function of the number of electrons, $\mathrm{N}$ i.e., functional of $\mathrm{N}, \mathrm{E}(\mathrm{N})$. Thereafter, following Iczkowski and Margrave [40], Parr et al. [41] defined the electronegativity as the additive inverse of the chemical potential

$\chi=-\mu$

or, $\chi=-[\partial \mathrm{E} / \partial \mathrm{N}]_{\mathrm{v}}$

The absolute hardness, $\eta$ of the density functional theory [42] is defined as

$\eta=1 / 2[\partial \mu / \partial \mathrm{N}]_{\mathrm{v}}=1 / 2\left[\left(\partial^{2} \mathrm{E} / \partial \mathrm{N}^{2}\right)\right]_{\mathrm{v}}$

Although mathematical formulae were suggested but rigorous evaluation of electronegativity, $\chi$ and absolute hardness $\eta$ in terms of the suggested formulae, Eq. (3) and Eq. (4), has not been possible. However, calculus of finite difference approximation was invoked to suggest approximate and operational formulae of electronegativity and hardness as under:

$\chi=(I+A) / 2$

$\eta=(\mathrm{I}-\mathrm{A}) / 2$

where ' $\mathrm{I}$ ' and ' $\mathrm{A}$ ' are the first ionization potential and electron affinity of the chemical species. Pearson [43] proceeded further to evaluate ' $\mathrm{I}$ ' and ' $\mathrm{A}$ ' in terms of orbital energies of the highest occupied molecular orbital, HOMO and the lowest unoccupied molecular orbital, LUMO by connecting it with Hartree-Fock SCF theory and invoking Koopmans' theorem

$\chi=\left(-\varepsilon_{\text {Hомо }}-\varepsilon_{\text {LUмо }}\right) / 2$

$\eta=\left(-\varepsilon_{\text {Hомо }}+\varepsilon_{\text {LUмо }}\right) / 2$

But the above finite difference approximation to $\chi$ and $\eta$ are not free from criticism and critical analysis has revealed the gross approximate nature of the above algorithms. We may point out that the rigorous theoretical calculation of hardness using the ansatz (4) is not easy because, numerical method is the only route of evaluating global hardness according to the Eq. (4). Sen and Vinayagam [44] evaluated the density functional hardness of 12 elements only and the true density functional global hardness of the majority of atoms are still at large. Moreover, Reed [45] pointed out that the invocation of the finite difference approximation requires that the absolute hardness be essentially independent of charge over an interval of two units of charge. This does not appear to be the case of chemical hardness or absolute hardness. We [46] also pointed out some inconsistency in the finite difference definition of hardness. Reed [45] has opined further that the operational definition contained in Eq. (6) and Eq. (8) does not just follow from the finite difference approximation. Thus, some inherent mathematical inconsistency in evaluating global hardness by finite difference approximation method is apparent. Although there are other formulae for the hardness and electronegativity when the quadratic model of Parr and Pearson is not valid [4749], the effort of density functional quantification of electronegativity and hardness is not that successful and these two descriptors are still qualitative per se.

Since we are after to reduce evidence that the basic nature of the electronegativity and the hardness are fundamentally qualitative per se and operationally the same entity, we may site similar views expressed by other workers within the purview of density functional theory. Pearson [50] suggested that for donor atoms, the electronegativity could be taken as a measure of the hardness of the base. Putz [51-54], after rigorous research on systematic formulation of 
electronegativity and hardness, opined out that the hardness and electronegativity are proportional to each other.

$$
\chi \propto \eta
$$

Ayers [38] in his effort to evaluate the electronegativity and hardness of neutral atoms on the basis of the energy expression of March and White [55], pointed out that the two fundamental atomic parameters, hardness and electronegativity, have the similar expression, more precisely, proportional to each other.

Recently, we have noticed another suggestion corrobo rating our view that the electronegativity and the hardness are two different appearances of the one and the same fundamental property of atoms and molecules. Li et al. [56] hold that "electronegativity represents the electron holding power of an atom; the stiffness of the atoms can thus be defined as electron holding energy of atoms per unit volume".

Now, let us have a bit Buddhist's contemplation over the subject that there is much commonality in the physical structure and the philosophical basis of hardness and electronegativity as regards their origin and operational significance. The hardness refers to the resistance of the electron cloud of the atomic and molecular systems under small perturbation of electrical field. An atom or molecule having least tendency of deformation are hard and having small tendency of deformation are soft. In other words, least polarizable means most hard and in such systems the electron clouds are tightly bound to the atoms or molecules. On the contrary most polarizable means least hard and in such systems the electron cloud is loosely bound to the atoms or molecules.

Electronegativity though defined in many different ways, the most logical and rational definition of it is the electron holding power of the atoms or molecules. The more electronegative species hold electrons more tightly and the less electronegative species hold less tightly [4].

Thus, if we invoke the qualitative definition of hardness stated above and compare with the qualitative definition of electronegativity, the commonality of their conceptual structures and philosophical basis are self-evident [4].

We are discovering the commonality in fundamental nature of hardness and electronegativity- the holding power of the electron cloud by the chemical species. Thus the qualitative views of the origins of hardness and electronegativity nicely converge to the one and single basic principle that they originate from the same source -the electron attracting power of the screened nuclear charge [4].

We have further points to note. A look at Eq. (5) - Eq. (8) reveals that one can calculate ' $\mathrm{I}$ ' and 'A' from SCF theory by invoking Koopmans' theorem. Again, although very accurate values of ' $\mathrm{I}$ ' are available, values of ' $\mathrm{A}$ ' are either very small tending to zero, or in most cases is unknown. It, therefore, transpires that if we neglect ' $\mathrm{A}$ ' and ignore the averaging factor, $1 / 2$ from Eq. (5) and Eq. (6), the simplified equations look,

$\chi=\mathrm{I}$

$\eta=I$

Thus, we see that the effort of quantification of the hardness and the electronegativity in terms of density functional theory degenerates to the give a grand equation declaring equality of $\chi, \eta$ and I.

$\chi=\eta=I$

Thus, both of them, $\chi$ and $\eta$ are numerically and dimensionally the same for atoms and molecules. Thus it is corroborated from the density functional theory also that the basic nature of $\chi$ and $\eta$ is the same [4]. We have pointed out the commonality in the basis of the physical structures of hardness and electronegativity in a recent work [4]. We have launched a series of search [57-58] whether our hypothesis that "the electronegativity and the hardness are two different appearances of the one and the same fundamental property of atoms" can be justified by application in the real world? We [57] have computed the internuclear distances of a number of diatomics as one descriptor of the real world on the basis of above hypothesis. We [58] have also applied our hypothesis to compute the charge distribution during the formation of molecules, i.e., the dipole charges and associated dipole moments of the molecules.

Since our hypothesis is that the origin and the operational significance of the electronegativity and hardness are the same, we [59-61] have also demonstrated that the equalization of hardness must occur during molecule formation similar to the well established physical process of the electronegativity equalization. We have demonstrated that if we pass a scale of hardness for a scale of electronegativity [4], the scale observes all empirical criteria of acceptability of a satisfactory electronegativity scale. But we have not tried yet whether a scale of electronegativity could be a true representative of a satisfactory scale of hardness.

We propose to perform a validity test of our hypothesis "The electronegativity and the hardness are two different appearances of the one and the same fundamental property of atoms" by considering well known scales of electronegativity as the scales of hardness. In this report we have invoked Gordy's [62] scale of electronegativity to pass for a scale of hardness.

\subsection{Gordy's scale of electronegativity}

In 1946, Gordy [59] defined the electronegativity ( $\chi$ ) of an atom as the electrostatic potential felt by the valence electrons at a radial distance equal to atom at the single bond covalent radius ( $r$ ). The atomic electronegativity ansatz of Gordy [59] is:

$\chi=\mathrm{e}\left(\mathrm{Z}_{\mathrm{eff}} / \mathrm{r}\right)$

In a recent work, Ghosh and Chakraborty [63] pointed out the prevalent dimensional mismatch in the Gordy's [62] atomic electronegativity ansatz and modified the ansatz by suggesting that the atomic electronegativity is not equal but proportional to $Z_{\text {eff }} / \mathrm{r}$ and also they replaced the covalent radius by the absolute radius in the Gordy's electronegativity ansatz:

$\chi=\mathrm{a}\left(\mathrm{Z}_{\text {eff }} / \mathrm{r}_{\mathrm{MP}}\right)+\mathrm{b}$

where $\mathrm{r}_{\mathrm{MP}}$ is the most probable radius of the atom and ' $a$ ' and ' $b$ ' are constants. They proposed and evaluated the value of ' $a$ ' and ' $b$ ' for each period separately.

\section{Method of computation}

Ghosh and Chakraborty [63] have revisited the electronegativity scale of Gordy [62] with the modifications noted above. Since the basic philosophy of the origin of hardness and electronegativity is the energy of attraction of screened nucleus upon valence electrons of atoms, we propose the ansatz of measurement of electronegativity, Eq. (14), as an ansatz of measuring the global hardness of atoms. Since the suggested ansatz of Ghosh and Chakraborty is $\chi=a\left(Z_{\text {eff }} / r_{M P}\right)+b$ for electronegativity, the hardness ansatz becomes, by our hypothesis,

$\eta=a\left(Z_{\text {eff }} / r\right)+b$ 
In determining ' $a$ ' and ' $b$ ' separately for each period, we have plotted the global hardness data published by us [46] against $\left(Z_{\text {eff }} / r\right)$ where $r$ is taken from the absolute radii values published by us [64]. It is pertinent to mention that we [61] have evaluated the absolute radii of atoms of 103 elements of periodic table assuming that the most probable radii of the outer most orbital of the atom is the atomic radius. Thus, both ansatz under reference, vide equations (14) and (15), compute the energy of attraction of the screened nucleus of the atom upon its valence electron. This is our basic definition of electronegativity and global hardness of atoms and hence their operational and scientific identity.

\section{Results and Discussion}

The least square fitted ' $a$ ' and ' $b$ ' are presented in Table 1. Now using the Eq. (16), we have evaluated the global hardness values of the atoms of 103 elements of the periodic table using the least square fitted ' $a$ ', ' $b$ ' and the absolute radii of all the 103 atoms published by us [64]. The global hardness data evaluated through the Eq. (15) are presented in Table 2. In order to explore whether the evaluated data exhibit periodicity we have plotted global hardness as a function of atomic number in Figure 1.

\begin{tabular}{lll}
\multicolumn{2}{l}{ Table 1. Computed a and b values. } \\
\hline Period & $\mathbf{a}$ & $\mathbf{b}$ \\
\hline First & 0.118 & 0.117 \\
Second & 0.034 & 0.102 \\
Third & 0.031 & 0.084 \\
Fourth & 0.031 & 0.089 \\
Fifth & 0.035 & 0.081 \\
Sixth & 0.01 & 0.054 \\
Seventh & 0.02 & 0.049 \\
\hline
\end{tabular}

Table 2. Comparative study of the sets of hardness data (in $\mathrm{eV}$ ) of the present calculation vis-à-vis Ghosh and Islam(GI), Putz, Pearson and Robles and Bartolotti (RB).

\begin{tabular}{|c|c|c|c|c|c|}
\hline Atom & $\eta$ Present work & $\eta \mathbf{G I}$ & $\eta$ Putz & $\eta$ Pearson & $\eta \mathbf{R B}$ \\
\hline $\mathrm{H}$ & 6.39435 & 6.429954 & 6.45 & 6.43 & 6.83 \\
\hline $\mathrm{He}$ & 12.46255 & 12.54491 & 25.79 & & 16.88 \\
\hline $\mathrm{Li}$ & 3.166293 & 2.374587 & 0.65 & 2.39 & 3.06 \\
\hline $\mathrm{Be}$ & 3.654912 & 3.496763 & 1.69 & 4.5 & 5.16 \\
\hline B & 4.33901 & 4.619009 & 3.46 & 4.01 & 4.39 \\
\hline $\mathrm{C}$ & 5.218454 & 5.740979 & 6.21 & 5 & 5.49 \\
\hline $\mathrm{N}$ & 6.293065 & 6.862467 & 9.59 & 7.23 & 8.59 \\
\hline 0 & 7.563914 & 7.985436 & 13.27 & 6.08 & 6.42 \\
\hline $\mathrm{F}$ & 9.029012 & 9.106475 & 16.16 & 7.01 & 7.52 \\
\hline $\mathrm{Ne}$ & 10.69158 & 10.23034 & 17.87 & & 15.45 \\
\hline $\mathrm{Na}$ & 2.739221 & 2.444141 & 0.66 & 2.3 & 2.91 \\
\hline $\mathrm{Mg}$ & 3.046934 & 3.014651 & 0.93 & 3.9 & 4.63 \\
\hline $\mathrm{Al}$ & 3.433784 & 3.584907 & 1.42 & 2.77 & 2.94 \\
\hline $\mathrm{Si}$ & 3.899699 & 4.155131 & 2.1 & 3.38 & 3.61 \\
\hline $\mathrm{P}$ & 4.445134 & 4.725804 & 2.92 & 4.88 & 5.42 \\
\hline S & 5.069535 & 5.295979 & 3.82 & 4.14 & 4.28 \\
\hline $\mathrm{Cl}$ & 5.772969 & 5.866186 & 5.01 & 4.68 & 4.91 \\
\hline $\mathrm{Ar}$ & 6.555909 & 6.436619 & 6.16 & & 10.69 \\
\hline $\mathrm{K}$ & 2.719916 & 2.327318 & 0.18 & 1.92 & 2.35 \\
\hline $\mathrm{Ca}$ & 2.922201 & 2.758724 & 0.25 & 4 & 3.07 \\
\hline $\mathrm{Sc}$ & 2.976222 & 2.858192 & 0.31 & 3.2 & 2.52 \\
\hline $\mathrm{Ti}$ & 3.033132 & 2.95783 & 0.38 & 3.37 & 2.03 \\
\hline V & 3.092708 & 3.057341 & 0.45 & 3.1 & \\
\hline $\mathrm{Cr}$ & 3.155012 & 3.156725 & 0.54 & 3.06 & 4.06 \\
\hline $\mathrm{Mn}$ & 3.220255 & 3.256383 & 0.64 & 3.72 & 2.88 \\
\hline $\mathrm{Fe}$ & 3.288155 & 3.355931 & 0.75 & 3.81 & 2.53 \\
\hline Co & 3.35896 & 3.455609 & 0.88 & 3.6 & 3.53 \\
\hline $\mathrm{Ni}$ & 3.432351 & 3.555013 & 1.02 & 3.25 & 4.08 \\
\hline $\mathrm{Cu}$ & 3.508508 & 3.654418 & 1.21 & 3.25 & \\
\hline $\mathrm{Zn}$ & 3.587666 & 3.75416 & 1.39 & 4.94 & 6.01 \\
\hline Ga & 3.962174 & 4.18552 & 1.59 & 2.9 & 3.03 \\
\hline Ge & 4.388494 & 4.616627 & 1.94 & 3.4 & 3.52 \\
\hline As & 4.877781 & 5.066215 & 2.35 & 4.5 & 5.04 \\
\hline Se & 5.397913 & 5.479496 & 2.87 & 3.87 & 3.95 \\
\hline $\mathrm{Br}$ & 5.980865 & 5.9111 & 3.39 & 4.22 & 4.4 \\
\hline $\mathrm{Kr}$ & 6.615076 & 6.341847 & 2.98 & & 9.45 \\
\hline $\mathrm{Rb}$ & 2.492098 & 2.120458 & 0.08 & 1.85 & 2.21 \\
\hline $\mathrm{Sr}$ & 2.687484 & 2.53737 & 0.11 & 3.7 & 3.08 \\
\hline
\end{tabular}

Table 2 (Continued).

\begin{tabular}{|c|c|c|c|c|c|}
\hline Atom & $\eta$ Present work & $\eta \mathbf{G I}$ & $\eta$ Putz & $\eta$ Pearson & $\eta \mathbf{R B}$ \\
\hline $\bar{Y}$ & 2.739707 & 2.633547 & 0.14 & 3.19 & 3.67 \\
\hline $\mathrm{Zr}$ & 2.794616 & 2.729753 & 0.17 & 3.21 & 2.09 \\
\hline $\mathrm{Nb}$ & 2.852208 & 2.825974 & 0.21 & 3 & 3.67 \\
\hline Mo & 2.912458 & 2.92213 & 0.25 & 3.1 & \\
\hline Tc & 2.975414 & 3.018371 & 0.29 & & 2.05 \\
\hline $\mathrm{Ru}$ & 3.041044 & 3.114598 & 0.35 & 3 & \\
\hline $\mathrm{Rh}$ & 3.109328 & 3.210756 & 0.41 & 3.16 & \\
\hline $\mathrm{Pd}$ & 3.180302 & 3.306947 & 0.47 & 3.89 & 6.32 \\
\hline $\mathrm{Ag}$ & 3.253976 & 3.403195 & 0.55 & 3.14 & 3.5 \\
\hline $\mathrm{Cd}$ & 3.330303 & 3.499376 & 0.63 & 4.66 & 5.35 \\
\hline In & 3.692092 & 3.916369 & 0.73 & 2.8 & 2.77 \\
\hline Sn & 4.104122 & 4.333233 & 0.88 & 3.05 & 3.15 \\
\hline $\mathrm{Sb}$ & 4.566433 & 4.750079 & 1.1 & 3.8 & 4.39 \\
\hline $\mathrm{Te}$ & 5.07907 & 5.166979 & 1.34 & 3.52 & 3.47 \\
\hline I & 5.64201 & 5.583887 & 1.62 & 3.69 & 3.81 \\
\hline $\mathrm{Xe}$ & 6.255325 & 6.000897 & 1.92 & & 8.23 \\
\hline Cs & 1.543995 & 0.682915 & & 1.71 & 1.98 \\
\hline $\mathrm{Ba}$ & 1.594643 & 0.920095 & & 2.9 & 2.16 \\
\hline $\mathrm{La}$ & 1.658281 & 1.157089 & & 2.6 & 2.46 \\
\hline $\mathrm{Ce}$ & 1.735003 & 1.394276 & & & 1.8 \\
\hline $\mathrm{Pr}$ & 1.824769 & 1.631473 & & & 1.11 \\
\hline $\mathrm{Nd}$ & 1.927489 & 1.868439 & & & 0.7 \\
\hline Pm & 2.043334 & 2.105658 & & & 0.33 \\
\hline $\mathrm{Sm}$ & 2.172118 & 2.342665 & & & 0.02 \\
\hline $\mathrm{Eu}$ & 2.314019 & 2.579815 & & & 2.42 \\
\hline Gd & 2.46898 & 2.817026 & & & -1.02 \\
\hline $\mathrm{Tb}$ & 2.636854 & 3.054037 & & & 1.36 \\
\hline Dy & 2.81786 & 3.291169 & & & 1.06 \\
\hline Ho & 3.011902 & 3.528297 & & & 0.78 \\
\hline $\mathrm{Er}$ & 3.21896 & 3.765525 & & & 0.54 \\
\hline $\mathrm{Tm}$ & 3.439031 & 4.002555 & & & 0.32 \\
\hline $\mathrm{Yb}$ & 3.672004 & 4.239478 & & & 3.27 \\
\hline $\mathrm{Lu}$ & 3.918174 & 4.476583 & & & 3.64 \\
\hline $\mathrm{Hf}$ & 4.169252 & 4.706522 & & 3 & 3.94 \\
\hline Та & 4.449567 & 4.950847 & & 3.79 & 1.75 \\
\hline W & 4.734833 & 5.187931 & & 3.58 & 1.23 \\
\hline $\operatorname{Re}$ & 5.033418 & 5.425608 & & 3.87 & 2.13 \\
\hline Os & 5.344247 & 5.661914 & & 3.8 & 1.72 \\
\hline Ir & 5.66934 & 5.900043 & & 3.8 & 1.27 \\
\hline $\mathrm{Pt}$ & 6.006359 & 6.136715 & & 3.5 & 3.5 \\
\hline $\mathrm{Au}$ & 6.357058 & 6.37413 & & 3.46 & 3.44 \\
\hline $\mathrm{Hg}$ & 6.719813 & 6.610266 & & 5.54 & 5.29 \\
\hline $\mathrm{Tl}$ & 1.854981 & 1.704349 & & 2.9 & 2.69 \\
\hline $\mathrm{Pb}$ & 1.961715 & 1.941353 & & 3.53 & 3.02 \\
\hline $\mathrm{Bi}$ & 2.081548 & 2.178492 & & 3.74 & 4.14 \\
\hline Po & 2.214475 & 2.415812 & & & 3.28 \\
\hline At & 2.360287 & 2.652778 & & & 3.57 \\
\hline $\mathrm{Rn}$ & 2.519247 & 2.889955 & & & 7.69 \\
\hline $\mathrm{Fr}$ & 1.475727 & 0.988253 & & & \\
\hline $\mathrm{Ra}$ & 1.572363 & 1.28195 & & & \\
\hline Ac & 1.5982 & 1.349725 & & & \\
\hline Th & 1.625368 & 1.417526 & & & \\
\hline $\mathrm{Pa}$ & 1.87748 & 1.936857 & & & \\
\hline $\mathrm{U}$ & 2.054492 & 2.230558 & & & \\
\hline $\mathrm{Np}$ & 2.256266 & 2.52412 & & & \\
\hline $\mathrm{Pu}$ & 2.674334 & 3.043613 & & & \\
\hline $\mathrm{Am}$ & 2.983382 & 3.416868 & & & \\
\hline $\mathrm{Cm}$ & 3.011 & 3.404984 & & & \\
\hline $\mathrm{Bk}$ & 3.56106 & 3.92442 & & & \\
\hline $\mathrm{Cf}$ & 3.906333 & 4.218081 & & & \\
\hline Es & 4.276498 & 4.511593 & & & \\
\hline $\mathrm{Fm}$ & 4.671389 & 4.805093 & & & \\
\hline Md & 5.09156 & 5.098982 & & & \\
\hline No & 5.536423 & 5.392605 & & & \\
\hline $\mathrm{Lr}$ & 5.642892 & 5.460699 & & & \\
\hline
\end{tabular}

The strength of any model is its ability to explain experimental observations. But we have noted that the atomic hardness is neither an experimentally observable quantity nor it can be evaluated quantum mechanically. We, therefore, in absence of any benchmark for absolute hardness data, rely upon our experience of hardness behavior goaded by periodic law, and a comparison with various sets of available hardness data evaluated by different workers as a validity test. We have presented the present evaluated data along with those published by us [46], Pearson [65], Putz [66], Robles and Bartolotti [67] in Table 2. In order to examine the nature of variation of such different sets of data vis-à-vis the result of 


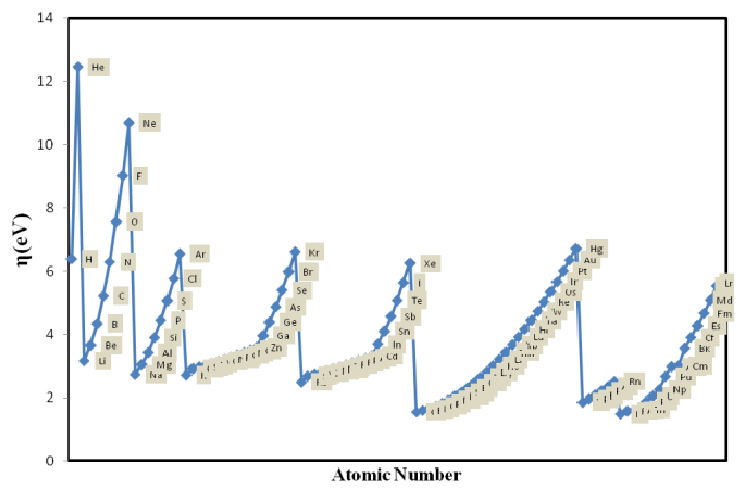

Figure 1. Plot of the computed hardness of 103 elements of the periodic table as a function of their atomic number.

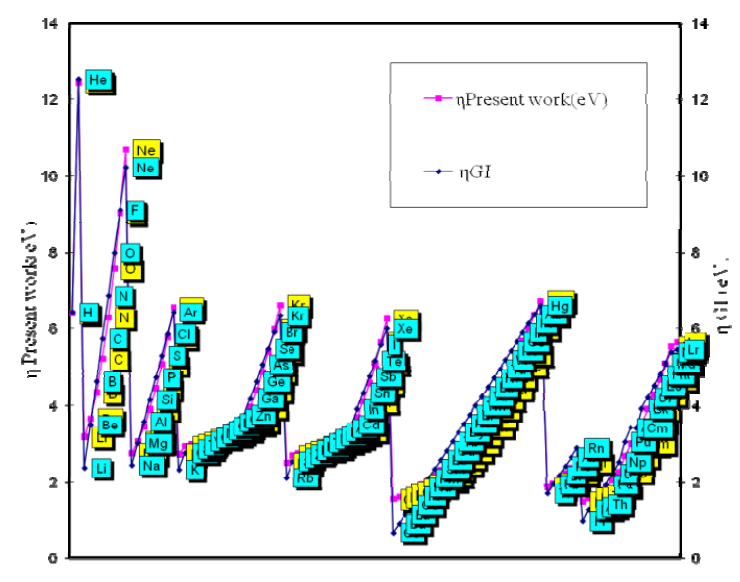

Figure 2. Comparative study of the computed hardness values vis-à-vis the hardness values computed by Ghosh and Islam.

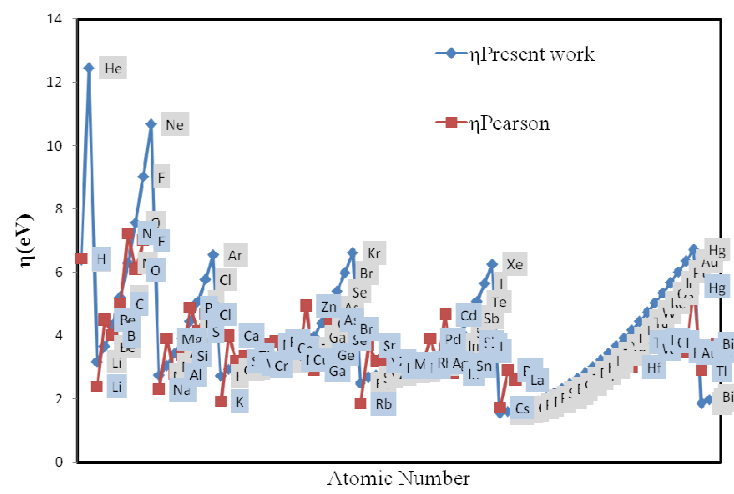

Figure 3. Comparative study of the computed hardness values vis-à-vis the hardness values computed by Pearson.

Table 3. Comparative study of the sets of hardness data of the present calculation vis-à-vis the DFT calculation of Sen and Vinayagam.

\begin{tabular}{lll}
\hline Atom & \multicolumn{1}{c}{$\boldsymbol{\eta}$ DFT } & $\boldsymbol{\eta}$ calculated \\
\hline $\mathrm{Li}$ & 2.33 & 3.166293 \\
$\mathrm{~B}$ & 4.07 & 4.33901 \\
$\mathrm{C}$ & 5 & 5.218454 \\
$\mathrm{~N}$ & 5.91 & 6.293065 \\
$\mathrm{O}$ & 6.8 & 7.563914 \\
$\mathrm{~F}$ & 7.66 & 9.029012 \\
$\mathrm{Na}$ & 2.23 & 2.739221 \\
$\mathrm{Al}$ & 2.69 & 3.433784 \\
$\mathrm{Si}$ & 3.33 & 3.899699 \\
$\mathrm{P}$ & 3.91 & 4.445134 \\
$\mathrm{~S}$ & 4.45 & 5.069535 \\
$\mathrm{Cl}$ & 4.98 & 5.772969 \\
\hline
\end{tabular}

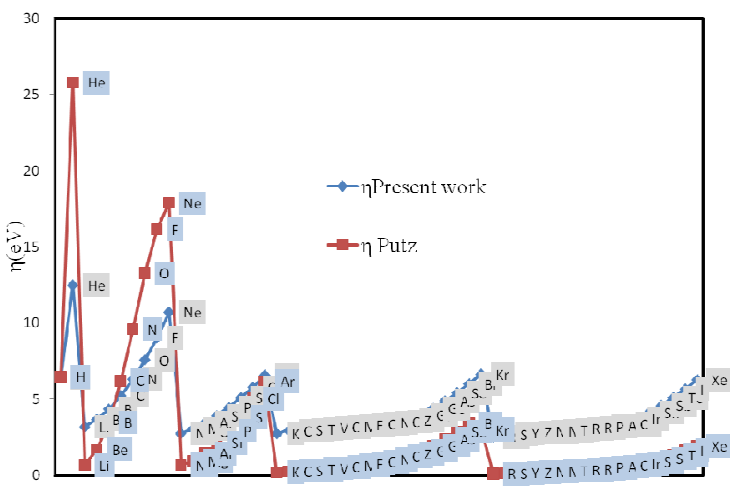

Atomi Number

Figure 4. Comparative study of the computed hardness values of 54 elements vis-à-vis the hardness values computed by Putz.

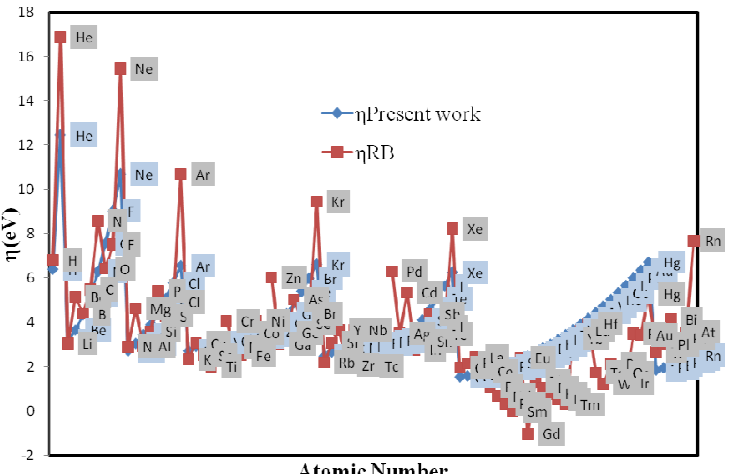

Figure 5. Comparative study of the computed hardness values of 86 elements vis-à-vis the corresponding hardness values of Robles and Bartolotti.

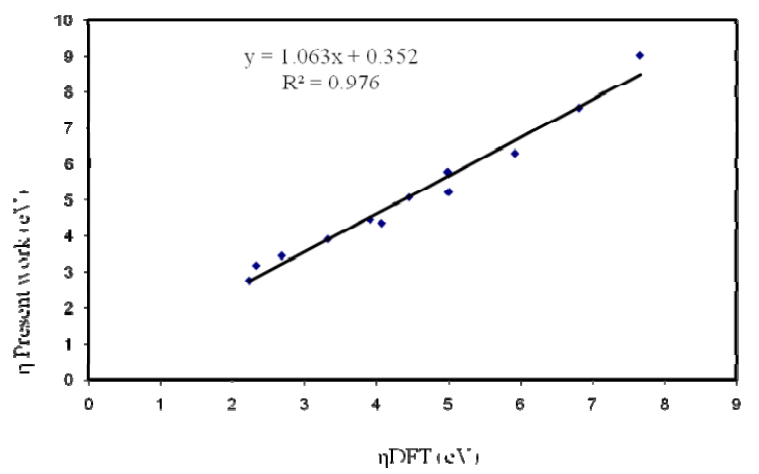

Figure 6. Comparative study of the computed hardness values vis-à-vis the DFT calculated hardness value of Sen and Vinayagam.

present venture, we have plotted the respective sets of data in Figures 2-5.

We have presented the result of the DFT calculation of Sen and Vinayagam [44] and the corresponding data of present calculation in Table 3 and plotted in Figure 6.

From Figure 1 and Table 2, it is evident that computed hardness values of the atoms of 103 elements exhibit perfect periodicity of periods and groups. Each period begins with the hardness of a representative element and ends with a noble gas atom. The hardness of the inert gas atoms occurs at the top of the curve. We note the following particular behavior of the computed hardness:

1. The computed hardness data of $\mathrm{N}, \mathrm{O}$, and $\mathrm{F}$ and also $\mathrm{P}, \mathrm{S}, \mathrm{Cl}$ follow the sequence $\mathrm{N}(6.293065)<0(7.563914)<\mathrm{F}$ $(9.029012)$, and $\mathrm{P}(4.445134)<\mathrm{S}(5.069535)<\mathrm{Cl}$ 
(5.772969). Thus, our results are similar to the results of Sen and Vinayagam [44]. It is further observed that the result of present calculation is free from the anomalous pattern of variation of the hardness values of these elements evident in the various finite difference approximation calculations [65,67].

2. It transpires from a comparative study of the numerical values of the hardness data in Table 3 that the results of present work have close agreement with the results of Sen and Vinayagam [44]. From Figure 6 it is more transparent that the results of the present calculation have close agreement, the correlation coefficient $\left(R^{2}\right)$ have the value 0.9762 , with a sophisticated DFT calculation. It is further revealed that the sequence of hardness data of the 12 elements in present work and that of Sen and Vinayagam [44] follow the same trend of variation with atomic number.

3. It is the rule of nature that high hardness means less deformability under small perturbation. It is also well known that the lanthanide elements are soft and easily deformable. It is well known that size of atoms of f-block elements undergo a steady but slow contraction and the effect is well reproduced in the radii of such elements. Pearson [65], relying upon the spatial behavior of f orbital, predicted that the hardness of the lanthanide elements should be small. It is distinct from Table 2 that the magnitude of hardness of all the lanthanide elements is small. Figure 1 reveals that the hardness values of lanthanide elements are close to each other and increases slowly with increasing atomic number.

4. It is distinct from Table 2 and Figure 1 that the global hardness values of the inert gas elements are highest in each period.

5. The global hardness values of Cs (1.543995) and Fr (1.475727) are significantly small compared to those of other elements. The strong chemical reactivity of the element Cs and Fr is well documented.

6. The chemical inertness of $\mathrm{Hg}$ and its state of aggregation is attributed to its small size and least deformability under small perturbation. Table 2 and Figure 1 reveal that the hardness value of Hg atom (6.719813) is quite high placing it in the group of inert gas elements. Thus, the present work can well correlate the significant and characteristic properties of elements in terms of its computed global hardness values.

7. A close examination of Table 2 and Figures 2-6 reveals that

(i) The nature of variations of the hardness of present calculation with atomic number vis-à-vis the corresponding nature of variation of our previously published data [46] is almost identical.

(ii) The nature of variation of the hardness of present calculation with atomic number vis-à-vis the corresponding nature of variation of Pearson's hardness [62] is quite comparable. The numerical values of hardness of a good number of elements in two sets of results are quite close.

(iii)The hardness values of Putz [66] and hardness values of present calculation have the similar trend of variation as a function of atomic number and both of them express periodicity.

(iv) It is surprising to note that, of the computed values of hardness by Robles and Bartolotti [67], the hardness of at least one element $(\mathrm{Gd})$ is negative $(-1.02)$ and hardly there is any periodic behaviour in the computed values of such global hardness.

\section{Conclusion}

In this work we have basically launched a further search whether our hypothesis that "the electronegativity and the hardness are two different appearances of the one and the same fundamental property of atoms" can be justified by using the electrostatic ansatz of measuring atomic electronegativity of Gordy currently modified by Ghosh and Chakraborty as an ansatz for measuring the global hardness of atoms. The ansatz under reference computes the energy of attraction of the screened nucleus of the atom upon its valence electron. This is our definition of electronegativity and global hardness of atoms. In this work we have computed the global hardness of the atoms of the103 elements of the periodic table using the Gordy's ansatz of measuring electronegativity, modified by Ghosh and Chakraborty, as the ansatz for measuring global hardness of atoms. We found the surprising results that the global hardnesses evaluated through an ansatz obtained by replacing electronegativity by hardness have fairly close agreement with other sets of available hardness data. The results of the present semi-empirical calculation also have strong correlation with the result of some sophisticated DFT calculation for a set of atoms. The express periodic behaviour and correlation of the most important physico-chemical properties of elements suggest that assumption that the electronegativity and the hardness are manifest two different descriptors of the one and the same fundamental property of atoms is justified.

\section{References}

[1]. Gilman, J. J. Mat. Res. Innovat. 1997, 1, 71-76

[2]. Pearson, R. G, J. Am. Chem. Soc. 1963, 85, 3533-3539

[3]. Pearson, R. G, Science 1966, 151, 172- 177.

[4]. Ghosh, D. C.; Islam, N., Int. J. Quantum Chem. 2009, DOI 10.1002/qua.22415 [Early view]

[5]. Pearson, R. G. J.Chem. Educ. 1999, 76, 267-274.

[6]. Pearson, R. G. J. Phys. Chem., 1994, 98, 1989-1992.

[7]. Nalewajski, R. F., J. Chem. Phys. 1983, 78, 6112-6120.

[8]. Yang, W.; Parr, R. G. Uytterhoeven, L. Phys. Chem. Miner. 1987, 15, 191-201.

[9]. Ayers P. W.: Yang, W. "Density Functional Theory", in Computational Medicinal Chemistry for Drug Discovery, Bultinck, P.; Winter, H. D.; Langenaeker, W.; Tollenaere, J. Eds., New York, Dekker, 2003, 571616.

[10]. Pritchard H. 0.; Skinner, H. A. Chem. Rev. 1955, 55, 745-786.

[11]. Ghosh, D. C. J. Indian Chem. Soc. 2003, 80, 527-533.

[12]. Coulson, C. A. Proc. R. Soc. London Ser. A 1951, 207, 63-73.

[13]. Fukui, K. Science 1982, 218, 747-754.

[14]. Lackner K. S.; Zweig, G. Phys. Rev. D 1983, 28, 1671-1691.

[15]. Klopman, G., J. Am. Chem. Soc. 1964, 86, 1463-1469

[16]. Klopman, G, J. Am. Chem. Soc. 1968, 90, 223-234.

[17]. Chermette, H. J. Comput. Chem. 1999, 20, 129-154.

[18]. Parr R. G.; Yang, W. T. Annu. Rev. Phys. Chem. 1995, 46, 701-728.

[19]. Gazquez, J. L. J. Mex. Chem. Soc. 2008, 52, 3-10.

[20]. Ayers, P. W.; Anderson, J. S. M; Bartolotti, L. J. Int. J. Quantum Chem 2005, 101, 520-534

[21]. Liu, S. B. Acta Phy. Chim. Sinica 2009, 25, 590-600.

[22]. Chattaraj, P. K.; Sarkar, U.; Roy, D. R. Chem. Rev. 2006, 106, 2065-2091.

[23]. Geerlings, P; Proft. F. D; Langenaeker, W. Chem. Rev. 2003, 103, 17931874

[24]. Pearson, R. G. J. Chem. Edu. 1987, 64, 561-567.

[25]. Pearson, R. G. Acc. Chem. Res. 1993, 26, 250-255

[26]. Chattaraj, P. K., Sengupta, S. J. Phys. Chem. 1996, 100, 16126-16130.

[27]. Zhou Z.; Parr, R. G. J. Am. Chem. Soc. 1989, 111, 7371-7379.

[28]. Parr R. G. ; Chattaraj, P.K. J. Am. Chem. Soc. 1991, 113, 1854-1855.

[29]. Chattaraj P. K.; Nath S.; Sannigrahi, A. B. J. Phys. Chem. 1994, 98, $9143-$ 9145.

[30]. Pearson, R. G.; Palke, W. E. J. Phys. Chem. 1992, 96, 3283-3285.

[31]. Pal, S.; Vaval N.; Roy, R. J. Phys. Chem. 1993, 97, 4404-4406.

[32]. Chattaraj, P. K. ; Liu G. H. ; Parr, R. G. Chem. Phys. Lett. 1995, 237, 171 176.

[33]. Ayers P. W.; Parr, R. G. J. Am. Chem. Soc. 2000, 122, 2010-2018.

[34]. Ghosh, D. C.; Jana, J. Bhattacharyya, S, Int. J. Quantum Chem. 2002, 87, 111-134.

[35]. Putz, M.V. Int. J. Quantum Chem. 2009, 109, 733-738.

[36]. Frenking G.; Krapp, A. J. Comput. Chem. 2007, 28, 15-24

[37]. Parr,R.G; Ayers, P W.; Nalewajski R. F.; J. Phys. Chem. A, 2005, 109, 3957-3959.

[38]. Ayers, P. W. Faraday Discuss, 2007, 135, 161-190.

[39]. Gyftpoulous, E. P.; Hatsopoulos, G. N. Proc. Natl. Acad. Sc. 1968, 60, 786-793.

[40]. Iczkowski, R. P.; Margrave, J. L. J. Am. Chem. Soc. 1961, 83, 3547-3551. 
[41]. Parr, R. G.; Donnelly, R. A.; Levy, M.; Palke, W. E. J. Chem. Phys. 1978, 68, 3801-3807.

[42]. Parr, R. G.; Pearson, R. G. J. Am. Chem. Soc. 1983, 105, 7512-7516

[43]. Pearson, R. G. Proc. Natl. Acad. Sci. 1986, 83, 8440-8441.

[44]. Sen K. D.; Vinayagam, S. C. Chem. Phys. Let. 1988, 144, 178-179.

[45]. Reed, J. L. J. Phys. Chem. A 1997, 101, 7396-7400.

[46]. Ghosh, D. C.; Islam, N., Int. J. Quantum Chem. 2009, 109, 110, 12061214.

[47]. Ayers, P. W.; Parr, R. G. J. Chem. Phys. 2008, 128, 184108(1)184108(8).

[48]. Parr, R. G; Bartolotti, L. J. J. Am. Chem. Soc. 1982, 104, 3801-3803.

[49]. Noorizadeh, S; Shakerzadeh, E. J. Phys. Chem. A 2008, 112, 3486-3491.

[50]. Pearson, R. G. Chem. Commun. 1968, 65-67.

[51]. Putz, M. V. Absolute and Chemical Electronegativity and Hardness, Nova Science Publishers, Inc., New York, 2008.

[52]. Putz, M. V.; Russo, N.; Sicilia, E. J. Comput. Chem. 2004, 25, 994-1003.

[53]. Putz, M.V.J. Theoret. Comput. Chem. 2007, 6, 33-47.

[54]. Putz, M.V. MATCH Commun. Math. Comput. Chem. 2008, 60, 845-868.

[55]. March, N. H; White, R. J. J. Phys. B 1972, 5, 466-475.

[56]. Li, K.; Wang, X.; Zhang,F.; Xue, D. Phys. Rev. Lett. 2008, 100, 235504(1)- 235504(4).

[57]. Ghosh; D. C.; Islam, N. Int. J. Quantum Chem., 2010, DOI: 10.1002/qua.22500, [Early View].

[58]. Ghosh; D. C.; Islam, N. Int. J. Quantum Chem., 2010, DOI: 10.1002/qua.22651 [Early View].

[59]. Ghosh; D. C.; Islam, N. Int. J. Quantum Chem., 2010, DOI: 10.1002/qua.22499, [Early View].

[60]. Ghosh; D. C.; Islam, N. Int. J. Quantum Chem., 2010 DOI: 10.1002/qua.22508, [Early View]

[61]. Ghosh; D. C.; Islam, N. Int. J. Quantum Chem., 2010, DOI: 10.1002/qua.22653 [Early View].

[62]. Gordy, W. Phys. Rev, 1946, 69, 604-607.

[63]. Ghosh, D. C.; Chakraborty, T. J. Mol. Str.-Theochem. 2009, 906, 87-93.

[64]. Ghosh, D. C; Biswas, R; Chakraborty, T; Islam, N; Rajak, S. K, J. Mol. Str.-Theochem. 2008, 865, 60-67.

[65]. Pearson, R. G. Inorg. Chem. 1988, 27, 734-740.

[66]. Putz, M. V. Int. J. Quantum. Chem. 2006, 106, 361-389.

[67]. Robles, J.; Bartolotti, L. J. J. Am. Chem. Soc. 1984, 106, 3723-3727. 